\title{
LHC benchmark scenarios for the real Higgs singlet extension of the standard model
}

\author{
Tania Robens ${ }^{1, \mathrm{a}}$, Tim Stefaniak ${ }^{2, \mathrm{~b}}$ \\ ${ }^{1}$ Institut für Kern- und Teilchenphysik, TU Dresden, Zellescher Weg 19, 01069 Dresden, Germany \\ ${ }^{2}$ Department of Physics and Santa Cruz Institute for Particle Physics, University of California, Santa Cruz, CA 95064, USA
}

Received: 20 February 2016 / Accepted: 26 April 2016/ Published online: 13 May 2016

(C) The Author(s) 2016. This article is published with open access at Springerlink.com

\begin{abstract}
We present benchmark scenarios for searches for an additional Higgs state in the real Higgs singlet extension of the Standard Model in Run 2 of the LHC. The scenarios are selected such that they fulfill all relevant current theoretical and experimental constraints, but can potentially be discovered at the current LHC run. We take into account the results presented in earlier work and update the experimental constraints from relevant LHC Higgs searches and signal rate measurements. The benchmark scenarios are given separately for the low-mass and high-mass region, i.e. the mass range where the additional Higgs state is lighter or heavier than the discovered Higgs state at around $125 \mathrm{GeV}$. They have also been presented in the framework of the LHC Higgs Cross Section Working Group.
\end{abstract}

\section{Introduction}

The first run of the LHC at center-of-mass (CM) energies of 7 and $8 \mathrm{TeV}$ has been completed in 2015. Its remarkable success is highlighted by the breakthrough discovery of a scalar boson in July 2012 and the measurements of its coupling properties, which thus far are well compatible with the interpretation in terms of the Higgs boson of the Standard Model (SM) Higgs mechanism [1-5]. The combination of the Higgs mass measurements performed by ATLAS and CMS yields [6]

$m_{H}=125.09 \pm 0.21$ (stat.) \pm 0.11 (syst.) $\mathrm{GeV}$.

If the discovered particle is indeed the Higgs boson of the SM, its mass measurement determines the last unknown ingredient of this model, as all other properties of the electroweak sector then follow directly from theory. In the coming years a thorough investigation of the Higgs boson's properties is

\footnotetext{
a e-mail: Tania.Robens@tu-dresden.de

be-mail: tistefan@ucsc.edu
}

needed in order to identify whether the SM Higgs sector is indeed complete, or instead, the structure of a more involved Higgs sector is realized. This includes detailed and accurate measurements of its coupling strengths and $\mathcal{C P}$ structure at the LHC and ultimately at future experimental facilities for Higgs boson precision studies. Complementary to this, collider searches for additional Higgs bosons need to be continued over the full accessible mass range. The discovery of another Higgs boson would inevitably prove the existence of a non-minimal Higgs sector.

In this work we consider the simplest extension of the SM Higgs sector, where an additional real scalar field is added, which is neutral under all quantum numbers of the SM gauge groups $[7,8]$ and acquires a vacuum expectation value (VEV). This model has been widely studied in the literature [9-52], also in the context of electroweak higher order corrections $[53,54]$ or offshell and interference effects $[33,34,55-59]$. Here, we present an update of the exploration of the model parameter space presented in Ref. [38], where we take the latest experimental constraints into account. As before, we consider masses of the second (non-standard) Higgs boson in the whole mass range up to $1 \mathrm{TeV}$. This minimal setup can be interpreted as a limiting case for more generic BSM scenarios, e.g. models with additional gauge sectors [60] or additional matter content [61,62]. Experimental searches for the model have been presented in [63-70].

As in Ref. [38] we take the following theoretical and experimental constraints into account: bounds from perturbative unitarity and electroweak (EW) precision measurements, in particular focussing on higher order corrections to the $W$ boson mass [32]; perturbativity, vacuum stability and correct minimization of the model up to a high energy scale using renormalization group (RG) evolved couplings; exclusion limits from Higgs searches at the LEP, Tevatron and LHC experiments via the public tool HiggsBounds [71-75], and compatibility of the model 
with the signal strength measurements of the discovered Higgs state using Higgs Signals [76] (cf. also Ref. [77]).

We separate the discussion of the parameter space into two different mass regions: (i) the high-mass region, $m_{H} \in$ $[130,1000] \mathrm{GeV}$, where the lighter Higgs boson $h$ is interpreted as the discovered Higgs state; (ii) the low-mass region, $m_{h} \in[1,120] \mathrm{GeV}$, where the heavier Higgs boson $H$ is interpreted as the discovered Higgs state.

We find that the most severe constraints in the whole parameter space for the second Higgs mass $m_{H} \lesssim 250 \mathrm{GeV}$ are mostly given by limits from collider searches for a SM Higgs boson as well as by the LHC Higgs boson signal strength measurements. For $m_{H} \gtrsim 250 \mathrm{GeV}$ limits from higher order contributions to the $W$ boson mass prevail, followed by the requirement of perturbativity of the couplings.

For the remaining viable parameter space we present predictions for signal cross sections of the yet undiscovered second Higgs boson for the LHC at a CM energy of $14 \mathrm{TeV}$, discussing both the SM Higgs decay signatures and the novel Higgs-to-Higgs decay mode $H \rightarrow h h$. For both the highmass and the low-mass regions we present a variety of benchmark scenarios. These are designed to render a maximal direct production rate for the collider signature of interest. Whenever kinematically accessible we give two different benchmark points for each mass, for which the Higgs-toHiggs decay $H \rightarrow h h$ is maximal or minimal, respectively.

The paper is organized as follows: In Sect. 2 we briefly review the model and the chosen parametrization. In Sect. 3 we review the constraints that are taken into account and in particular discuss the impact of the new constraints on the parameter space. In Sect. 4 we provide benchmark points and planes discussed above. We summarize and conclude in Sect. 5.

\section{The model}

In the following we briefly review the main features of the real Higgs singlet extension of the SM that are important for the benchmark choices. More details as regards the model can e.g. be found in Refs. $[29,32,38,54]$ and references therein.

\subsection{Potential and couplings}

The real Higgs singlet extension of the SM $[7,8,78]$ contains a complex $S U(2)_{L}$ doublet, in the following denoted by $\Phi$, and in additional a real scalar $S$ which is a singlet under the SM gauge group. The most general renormalizable Lagrangian compatible with an additional $Z_{2}$ symmetry is then given by

$\mathscr{L}_{s}=\left(D^{\mu} \Phi\right)^{\dagger} D_{\mu} \Phi+\partial^{\mu} S \partial_{\mu} S-V(\Phi, S)$,

with the scalar potential

$$
\begin{aligned}
V(\Phi, S)= & -m^{2} \Phi^{\dagger} \Phi-\mu^{2} S^{2} \\
& +\left(\Phi^{\dagger} \Phi S^{2}\right)\left(\begin{array}{cc}
\lambda_{1} & \frac{\lambda_{3}}{2} \\
\frac{\lambda_{3}}{2} \lambda_{2}
\end{array}\right)\left(\begin{array}{c}
\Phi^{\dagger} \Phi \\
S^{2}
\end{array}\right) \\
= & -m^{2} \Phi^{\dagger} \Phi-\mu^{2} S^{2}+\lambda_{1}\left(\Phi^{\dagger} \Phi\right)^{2}+\lambda_{2} S^{4} \\
& +\lambda_{3} \Phi^{\dagger} \Phi S^{2} .
\end{aligned}
$$

The implicitly imposed $Z_{2}$ symmetry forbids all linear or cubic terms of the singlet field $S$ in the potential. We assume that both Higgs fields $\Phi$ and $S$ have a non-zero vacuum expectation value (VEV), denoted by $v$ and $x$, respectively. In the unitary gauge, the Higgs fields are given by

$\Phi \equiv\left(\begin{array}{c}0 \\ \frac{\tilde{h}+v}{\sqrt{2}}\end{array}\right), \quad S \equiv \frac{h^{\prime}+x}{\sqrt{2}}$.

After diagonalization of the mass matrix we obtain the mass eigenstates $h$ and $H$ with mass eigenvalues given by

$m_{h}^{2}=\lambda_{1} v^{2}+\lambda_{2} x^{2}-\sqrt{\left(\lambda_{1} v^{2}-\lambda_{2} x^{2}\right)^{2}+\left(\lambda_{3} x v\right)^{2}}$,

$m_{H}^{2}=\lambda_{1} v^{2}+\lambda_{2} x^{2}+\sqrt{\left(\lambda_{1} v^{2}-\lambda_{2} x^{2}\right)^{2}+\left(\lambda_{3} x v\right)^{2}}$,

and $m_{h}^{2} \leq m_{H}^{2}$ by convention. The gauge and mass eigenstates are related via the mixing matrix

$$
\left(\begin{array}{c}
h \\
H
\end{array}\right)=\left(\begin{array}{cc}
\cos \alpha & -\sin \alpha \\
\sin \alpha & \cos \alpha
\end{array}\right)\left(\begin{array}{c}
\tilde{h} \\
h^{\prime}
\end{array}\right),
$$

where the mixing angle $-\frac{\pi}{2} \leq \alpha \leq \frac{\pi}{2}$ is given by

$$
\begin{aligned}
\sin 2 \alpha & =\frac{\lambda_{3} x v}{\sqrt{\left(\lambda_{1} v^{2}-\lambda_{2} x^{2}\right)^{2}+\left(\lambda_{3} x v\right)^{2}}}, \\
\cos 2 \alpha & =\frac{\lambda_{2} x^{2}-\lambda_{1} v^{2}}{\sqrt{\left(\lambda_{1} v^{2}-\lambda_{2} x^{2}\right)^{2}+\left(\lambda_{3} x v\right)^{2}}} .
\end{aligned}
$$

It follows from Eq. (7) that the light (heavy) Higgs boson couplings to SM particles are suppressed by $\cos \alpha(\sin \alpha)$.

If kinematically allowed, the additional decay channel $H \rightarrow h h$ is present. Its partial decay width at leading order (LO) is given by $[7,78]$

$\Gamma_{H \rightarrow h h}=\frac{\left|\mu^{\prime}\right|^{2}}{8 \pi m_{H}} \sqrt{1-\frac{4 m_{h}^{2}}{m_{H}^{2}}}$,

where the coupling strength $\mu^{\prime}$ of the $H \rightarrow h h$ decay reads

$\mu^{\prime}=-\frac{\sin (2 \alpha)}{2 v x}(\sin \alpha v+\cos \alpha x)\left(m_{h}^{2}+\frac{m_{H}^{2}}{2}\right)$.

Next-to-leading order (NLO) corrections to the $H \rightarrow h h$ decay width for this model have been calculated recently in Ref. [54]. The branching ratios of the heavy Higgs mass eigenstate $m_{H}$ are then given by

$$
\begin{aligned}
\mathrm{BR}_{H \rightarrow h h} & =\frac{\Gamma_{H \rightarrow h h}}{\Gamma_{\mathrm{tot}}}, \\
\mathrm{BR}_{H \rightarrow \mathrm{SM}} & =\sin ^{2} \alpha \times \frac{\Gamma_{\mathrm{SM}, H \rightarrow \mathrm{SM}}}{\Gamma_{\text {tot }}},
\end{aligned}
$$


where $\Gamma_{\mathrm{SM}} H \rightarrow \mathrm{SM}$ is the partial decay width of the SM Higgs boson and $H \rightarrow$ SM represents any SM Higgs decay mode. The total width is then

$\Gamma_{\text {tot }}=\sin ^{2} \alpha \times \Gamma_{\text {SM,tot }}+\Gamma_{H \rightarrow h h}$,

where $\Gamma_{\mathrm{SM}}$, tot denotes the total width of the SM Higgs boson with mass $m_{H}$. The suppression by $\sin ^{2} \alpha$ directly follows from the suppression of all SM-like couplings, cf. Eq. (7). For $\mu^{\prime}=0$, the decay $H \rightarrow h h$ vanishes and we recover the SM Higgs boson branching ratios.

For the collider phenomenology of the model two features are important:

- the suppression of the production cross section of the two Higgs states induced by the mixing, which is given by $\sin ^{2} \alpha\left(\cos ^{2} \alpha\right)$ for the heavy (light) Higgs, respectively;

- the suppression of the Higgs decay modes to SM particles, which is realized if the competing decay mode $H \rightarrow h h$ is kinematically accessible.

For the high-mass (low-mass) scenario, i.e. the case where the light (heavy) Higgs boson is identified with the discovered Higgs state at $\sim 125 \mathrm{GeV},|\sin \alpha|=0$ (1) corresponds to the complete decoupling of the second Higgs boson and therefore the SM-like scenario.

\subsection{Model parameters}

At the Lagrangian level, the model has five free parameters,

$\lambda_{1}, \lambda_{2}, \lambda_{3}, v, x$

while the values of the additional parameters $\mu^{2}, m^{2}$ are fixed by the minimization conditions. A more intuitive basis, where the free model parameters are represented by physical (i.e. observable) quantities, is given by ${ }^{1}$

$m_{h}, m_{H}, \sin \alpha, v, \tan \beta \equiv \frac{v}{x}$.

The vacuum expectation value of the Higgs doublet $\Phi$ is given by the SM value $v \sim 246 \mathrm{GeV}$, and one of the Higgs masses is fixed to $m_{h / H}=125.09 \mathrm{GeV}$, eliminating two of the five parameters. We are thus left with only three independent parameters,

$\left\{m \equiv m_{H / h}, \sin \alpha, \tan \beta\right\}$,

where the latter enters the collider phenomenology only through the heavy Higgs decay mode into the lighter Higgs, $H \rightarrow h h$. Note that from a collider perspective, for cases where the decay mode $H \rightarrow h h$ is kinematically allowed,

\footnotetext{
${ }^{1}$ Note that even if the $Z_{2}$ symmetry is not imposed, the parameters of the model relevant for the collider phenomenology considered here can always be chosen in terms of the masses, a mixing angle, and an additional parameter determining the $H \rightarrow h h$ decay channel.
}

the input parameter $\tan \beta$ could be replaced by either the total width of the heavier state, $\Gamma(H)$, the branching ratio $\mathrm{BR}(H \rightarrow h h)$, or the partial decay width of this channel, $\Gamma(H \rightarrow h h)$, respectively, rendering the following viable parameter choices besides Eq. (17):

$$
\begin{aligned}
& \left\{m \equiv m_{H / h}, \sin \alpha, \Gamma(H)\right\}, \\
& \left\{m \equiv m_{H / h}, \sin \alpha, \operatorname{BR}(H \rightarrow h h)\right\}, \\
& \left\{m \equiv m_{H / h}, \sin \alpha, \Gamma(H \rightarrow h h)\right\} .
\end{aligned}
$$

If the insertion starts on the Lagrangian level (via e.g. FeynRules [79], SARAH [80,81] or similar), also the Lagrangian parameters as such can be used as input values, but then care must be taken to correctly translate these into the phenomenologically viable parameter regions.

\section{Constraints}

In this section we list all theoretical and experimental constraints that we take into account, and give an overview over the impact of these constraints on the parameter space. We refer the reader to Ref. [38] for details of the implementation of these constraints. With respect to Ref. [38] we update the experimental limits from LHC Higgs searches, leading to a change in the allowed parameter space especially in the lower mass range, $m_{H} \in[130,250] \mathrm{GeV}$. We also include constraints from the combined ATLAS and CMS Higgs signal strength [82], rendering a significantly stronger limit on the mixing angle. However, this limit is still not as strong as the constraint from the $W$ boson mass measurement in most of the parameter space.

\subsection{Theoretical constraints}

We consider the following theoretical constraints in the selection of the benchmark scenarios:

- vacuum stability and minimization of model up to a scale $\mu_{\text {run }}=4 \times 10^{10} \mathrm{GeV}$

- perturbative unitarity of the $2 \rightarrow 2 S$-matrix for $\left(W^{+} W^{-}, Z Z, h h, h H, H H\right)$ initial and final states,

- perturbativity of the couplings in the potential, $\left|\lambda_{i}\right| \leq$ $4 \pi$, up to a high energy scale, $\mu_{\text {run }}=4 \times 10^{10} \mathrm{GeV}$, employing one-loop renormalization group equations (RGEs) [83].

\subsection{Experimental constraints}

The following experimental constraints are taken into account at the $95 \%$ C.L.: 
- agreement with electroweak precision observables, employing the oblique parameters $S, T, U$ [84-87] and using the results from the global fit from the GFitter Group [88],

- agreement with the observed $W$ boson mass [89-91], $M_{W}=80.385 \pm 0.015 \mathrm{GeV}$, employing the NLO calculation presented in Ref. [32],

- agreement with limits from direct Higgs searches at LEP, Tevatron, and the LHC using HiggsBounds (version 4.3.1) [71-75]. With respect to the results presented in Ref. [38], limits from the following searches have been included here:

- ATLAS search for $H \rightarrow W W$ [92],

- ATLAS search for $H \rightarrow Z Z$ [70],

- combination of ATLAS searches for $H \rightarrow h h \rightarrow$ $b b \tau \tau, \gamma \gamma W W^{*}, \gamma \gamma b b, b b b b[67]$,

- CMS search for $H \rightarrow V V\left(V=W^{ \pm}, Z\right)$ [66],

- CMS search for $H \rightarrow h h \rightarrow 4 \tau$, where $H$ is the SM-like Higgs boson at $125 \mathrm{GeV}$ [93].

- agreement with the observed signal strengths of the $125 \mathrm{GeV}$ Higgs boson, using HiggsSignals (version 1.4.0) [76], and using the results from the ATLAS and CMS combination of the LHC Run 1 data, $\mu=$ $1.09 \pm 0.11$ [82], leading to

$|\sin \alpha| \leq 0.36$

for the heavy Higgs mass range $m_{H} \gtrsim 150 \mathrm{GeV}$ (highmass range, $m_{h} \sim 125 \mathrm{GeV}$ ), and

$|\sin \alpha| \geq 0.87$ for the light Higgs mass range $m_{h} \lesssim 100 \mathrm{GeV}$ (low-mass range, $\left.m_{H} \sim 125 \mathrm{GeV}\right)$. In these mass regions potential signal overlap with the SM-like Higgs at $125 \mathrm{GeV}$ can be neglected. For Higgs masses in the range [100, 150] GeV we employ HiggsSignals using observables from the individual Higgs channels, which enables to approximately take into account a potential signal overlap [76], see also Ref. [38] for details.

\subsection{Allowed parameter regions and sensitivity} of the constraints

\subsubsection{High-mass region}

The importance of the different constraints on the mixing angle $\sin \alpha$ in the high-mass region, where $m_{h} \sim 125 \mathrm{GeV}$, is summarized in Fig. 1. Recall that this angle is responsible for the global suppression of the production cross section with respect to the SM prediction at the same Higgs mass. We see that in the lower mass region, $m_{H} \lesssim 250 \mathrm{GeV}$, the most important constraints stem from direct Higgs searches [66,70,94-96] and the combined Higgs signal strength [82], whereas for higher masses, $m_{H} \in[250 \mathrm{GeV} ; 800 \mathrm{GeV}]$, the $W$ boson mass becomes the strongest constraint [32]. Requiring perturbativity of the couplings yields the upper limit on $|\sin \alpha|$ for very heavy Higgs bosons, $m_{H} \geq 800 \mathrm{GeV}$.

The updated combined signal strength reduces the maximally allowed mixing angle from previously $|\sin \alpha| \lesssim 0.50$ [38] to $|\sin \alpha| \lesssim 0.36$. The updated limits from LHC Higgs searches in channels with vector boson final states also generally lead to stronger constraints, except in the region $m_{H} \in[260,300] \mathrm{GeV}$, where a statistical upward fluctua-

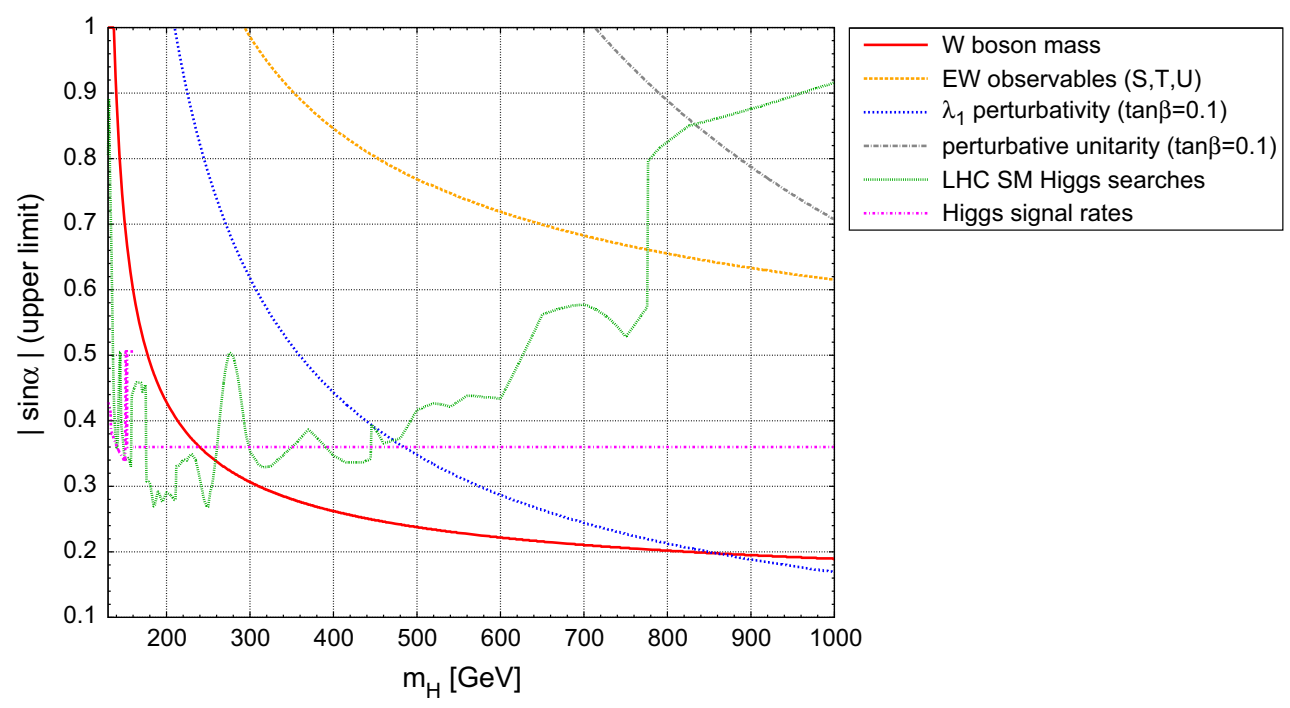

Fig. 1 Maximal allowed values for $|\sin \alpha|$ in the high-mass region, $m_{H} \in[130,1000] \mathrm{GeV}$, from NLO calculations of the $W$ boson mass (red, solid) [32], electroweak precision observables (EWPOs) tested via the oblique parameters $S, T$, and $U$ (orange, dashed), perturbativity of the RG-evolved coupling $\lambda_{1}$ (blue, dotted), evaluated for an exemplary choice $\tan \beta=0.1$, perturbative unitarity (gray, dash-dotted), direct LHC Higgs searches (green, dashed), and the Higgs signal strength (magenta, dash-dotted) 


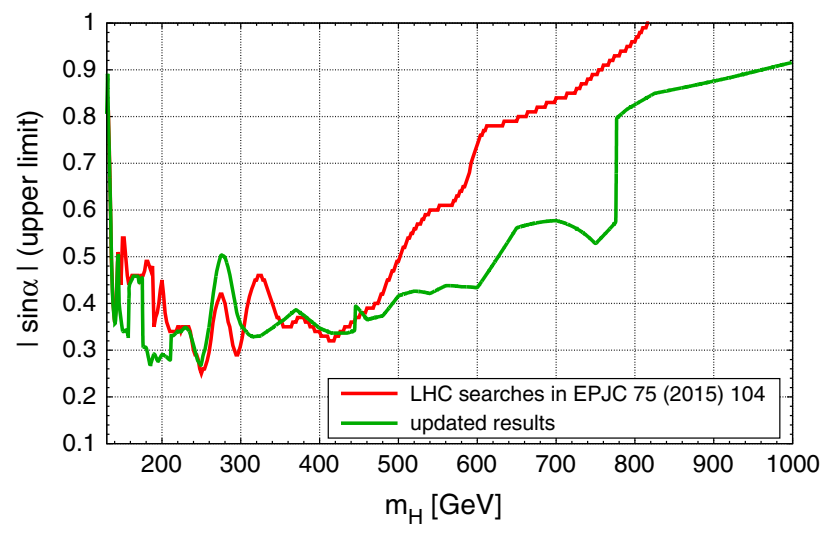

Fig. 2 Comparison of the $|\sin \alpha|$ limit obtained from the LHC Higgs searches with SM final states as presented in Ref. [38] (red) with the updated analysis (green)

Table 1 List of LHC Higgs search channels that are applied by HiggsBounds in the high-mass region, yielding the upper limit on $|\sin \alpha|$ shown in Figs. 1 and 2

\begin{tabular}{|c|c|c|}
\hline Range of $m_{H}$ [GeV] & Search channel & Reference \\
\hline $130-145$ & $\mathrm{H} \rightarrow \mathrm{ZZ} \rightarrow 41$ & [94] (CMS) \\
\hline $145-158$ & $\mathrm{H} \rightarrow \mathrm{VV}(\mathrm{V}=\mathrm{W}, \mathrm{Z})$ & [66] (CMS) \\
\hline $158-163$ & SM comb. & [95] (CMS) \\
\hline $163-170$ & $\mathrm{H} \rightarrow \mathrm{WW}$ & [96] (CMS) \\
\hline $170-176$ & SM comb. & [95] (CMS) \\
\hline $176-211$ & $\mathrm{H} \rightarrow \mathrm{VV}(\mathrm{V}=\mathrm{W}, \mathrm{Z})$ & [66] (CMS) \\
\hline $211-225$ & $\mathrm{H} \rightarrow \mathrm{ZZ} \rightarrow 41$ & [94] (CMS) \\
\hline $225-445$ & $\mathrm{H} \rightarrow \mathrm{VV}(\mathrm{V}=\mathrm{W}, \mathrm{Z})$ & [66] (CMS) \\
\hline $445-776$ & $\mathrm{H} \rightarrow \mathrm{ZZ}$ & [70] (ATLAS) \\
\hline $776-1000$ & $\mathrm{H} \rightarrow \mathrm{VV}(\mathrm{V}=\mathrm{W}, \mathrm{Z})$ & [66] (CMS) \\
\hline
\end{tabular}

tion in the CMS $H \rightarrow Z Z \rightarrow 4 \ell$ channel [66] leads to a slightly weaker limit than previously observed. A comparison of previously presented limits from LHC Higgs searches with the current status is displayed in Fig. 2. We see that the updated constraints yield stronger limits in particular for $m_{H} \leq 250 \mathrm{GeV}$ as well as for $m_{H} \gtrsim 400 \mathrm{GeV}$. We supplement this comparison by giving a detailed list in Table 1 of the LHC Higgs search channels that have been applied by HiggsBounds in the various mass regions. ${ }^{2}$

The relatively strong constraints on the mixing angle lead to a significant suppression of the direct production rates of the heavy Higgs boson at LHC run 2. Figure 3 shows the predicted production cross section at $14 \mathrm{TeV}$ after all con-

\footnotetext{
2 HiggsBounds selects the most sensitive channel by comparing the expected exclusion limits first. In a second step, the predicted signal strength is confronted with the observed exclusion limit only of this selected channel. This well-defined statistical procedure allows one to systematically test the model against a plethora of Higgs search limits without diluting the $95 \%$ C.L. of the individual limits.
}

straints have been taken into account. The production cross sections rapidly decrease with higher masses $m_{H}$ due to both the stronger constraints on the mixing angle (cf. Fig. 1) and a reduction of the available phase space for higher masses. The cross section for direct production in gluon fusion and successive decay into SM final states ranges from about $10 \mathrm{pb}$ at lower masses to about $10 \mathrm{fb}$ for masses around $800 \mathrm{GeV}$. Note that in order to obtain the predictions for a particular SM decay mode, $H \rightarrow X X$, these numbers need to be multiplied by a factor of $\mathrm{BR}(H \rightarrow X X) / \mathrm{BR}(H \rightarrow \mathrm{SM})$, where $\mathrm{BR}(H \rightarrow \mathrm{SM})$ is the sum over all branching ratios of Higgs decays into SM particles according to Eq. (13). Taking into account the current design strategy for the LHC run (cf. e.g. Ref. [97]) and expecting an integrated luminosity of about 100 and $300 \mathrm{fb}^{-1}$ before the shutdowns in 2019 and 2023, respectively, this translates into the fact that at least $\mathcal{O}\left(10^{3}\right)$ heavy Higgs bosons could be produced in that mass range in optimistic scenarios. For the $h h$ final state, on the other hand, cross sections are about an order of magnitude lower. A comparison of current exclusion limits from LHC $H \rightarrow h h$ searches with the predictions in the viable parameter space will be given in Sect. 4 .

Note that these plots were obtained using a simple rescaling of production cross section of a SM Higgs boson of the same mass as given in Ref. [23], i.e. contributions due to interference with the additional scalar are not included. Tools which can handle these have been presented e.g. in Refs. [55,56,58,59]. These studies, however, focus on effects on the line-shape of the heavy scalar boson after a possible discovery. Moreover, thus far, their calculations neglect additional higher order corrections, whereas these have been calculated to great precision for the SM Higgs boson and are included in Fig. 3 [23]. For the future, it would be desirable to perform a dedicated study of interference effects including higher order corrections for the benchmark points presented in this work in order to estimate their effects (and the systematic uncertainty introduced here by neglecting them).

\subsubsection{Low-mass region}

In the low-mass region, where the heavier Higgs state takes the role of the discovered Higgs boson, $m_{H} \sim 125 \mathrm{GeV}$, the parameter space is extremely constrained by the Higgs signal strength and exclusion limits from LEP Higgs searches [89]. The updated experimental results do not change the limits presented in Ref. [38]. We review these limits in Table 2. Note that in the low-mass region the couplings of the heavy Higgs boson at $125 \mathrm{GeV}$ become SM-like for $|\sin \alpha|=1$.

Table 3 gives the direct production cross section in gluon fusion for the undiscovered light Higgs state at a 8 and 14 $\mathrm{TeV}$ LHC, respectively. Again, the production cross section stems from a simple rescaling of the corresponding cross section for a SM Higgs boson of that mass [23,98]. 


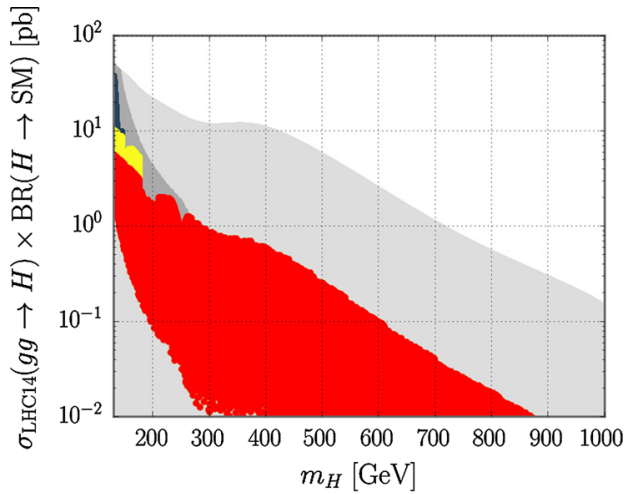

(a) Heavy Higgs signal rate with SM particles in the (b) final state for the LHC at $14 \mathrm{TeV}$.

Fig. 3 LHC signal rates of the heavy Higgs boson $H$ decaying into SM particles (a) or into two light Higgs bosons, $H \rightarrow h h$, (b), in dependence of the heavy Higgs mass, $m_{H}$, for a center-of-mass (CM) energy of $14 \mathrm{TeV}$. Shown are regions which are still allowed after all constraints are taken into account: Red and yellow regions correspond to agreement with the Higgs signal strength measurements at the $1 \sigma$

Table 2 Limits on $\sin \alpha$ and $\tan \beta$ in the low-mass scenario for various light Higgs masses $m_{h}$ and $\tan \beta=1$. In the second column we give the lower limit on $\sin \alpha$ stemming from exclusion limits from LEP or LHC Higgs searches (evaluated with HiggsBounds). If the lower limit on $\sin \alpha$ obtained from the Higgs signal rates (evaluated with HiggsSignals) results in stricter limits, they are displayed in the third column. The fourth column displays the upper limit on $\tan \beta$ that stems from perturbative unitarity in the complete decoupling case ( $|\sin \alpha|=1)$. In the fifth column we give the $\tan \beta$ value for which $\Gamma_{H \rightarrow h h}=0$ is obtained given the maximal mixing angle allowed by the Higgs exclusion limits (second column). At this $\tan \beta$ value, the $|\sin \alpha|$ limit obtained from the Higgs signal rates (third column) is abrogated. The table is taken from Ref. [38]

\begin{tabular}{lllcl}
\hline$m_{h}[\mathrm{GeV}]$ & $|\sin \alpha|_{\min , \mathrm{HB}}$ & $|\sin \alpha|_{\min , \mathrm{HS}}$ & $(\tan \beta)_{\max }$ & $(\tan \beta)_{\text {no } H \rightarrow h h}$ \\
\hline 120 & 0.410 & 0.918 & 8.4 & - \\
110 & 0.819 & 0.932 & 9.3 & - \\
100 & 0.852 & 0.891 & 10.1 & - \\
90 & 0.901 & - & 11.2 & - \\
80 & 0.974 & - & 12.6 & - \\
70 & 0.985 & - & 14.4 & - \\
60 & 0.978 & 0.996 & 16.8 & 0.21 \\
50 & 0.981 & 0.998 & 20.2 & 0.20 \\
40 & 0.984 & 0.998 & 25.2 & 0.18 \\
30 & 0.988 & 0.998 & 33.6 & 0.16 \\
20 & 0.993 & 0.998 & 50.4 & 0.12 \\
10 & 0.997 & 0.998 & 100.8 & 0.08 \\
\hline
\end{tabular}

\subsubsection{Intermediate mass region}

The intermediate mass region, where both Higgs bosons have masses between 120 and $130 \mathrm{GeV}$, was originally discussed in Ref. [38]. In this mass region the observed Higgs signal at

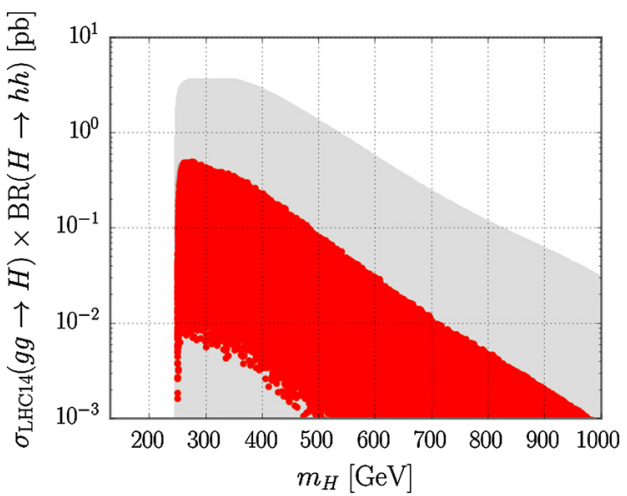

(b) Heavy Higgs signal rate with light Higgs bosons in the final state for the LHC at $14 \mathrm{TeV}$.

and $2 \sigma$ level, respectively, blue points comply with direct experimental searches but do not agree with the Higgs signal strength within $2 \sigma$. Light gray points denote scan points that are excluded by either perturbative unitarity, perturbativity of the couplings, RGE running or the $W$ boson mass, while dark gray points denote regions in parameter space that obey these constraints but are excluded by direct searches

Table 3 Maximally allowed cross section for light Higgs production in gluon fusion, $\sigma_{g g}=\left(\cos ^{2} \alpha\right)_{\max } \times \sigma_{g g, \text { SM }}$, at the LHC at CM energies of 8 and $14 \mathrm{TeV}$ after all current constraints have been taken into account, corresponding to the mixing angles from Table 2. This is an updated version of Tab. V in Ref. [38]

\begin{tabular}{llrlcc}
\hline$m_{h}[\mathrm{GeV}]$ & $\sigma_{g g}^{8 \mathrm{TeV}}[\mathrm{pb}]$ & $\sigma_{g g}^{14 \mathrm{TeV}_{[}}[\mathrm{pb}]$ & $m_{h}[\mathrm{GeV}]$ & $\sigma_{g g}^{8 \mathrm{TeV}_{[}}[\mathrm{pb}]$ & $\sigma_{g g}^{14 \mathrm{TeV}}[\mathrm{pb}]$ \\
\hline 120 & 3.28 & 8.41 & 60 & 0.63 & 1.38 \\
110 & 3.24 & 8.17 & 50 & 0.45 & 0.96 \\
100 & 6.12 & 15.10 & 40 & 0.76 & 1.59 \\
90 & 6.82 & 16.47 & 30 & 1.60 & 3.09 \\
80 & 2.33 & 5.41 & 20 & 5.04 & 8.97 \\
70 & 1.72 & 3.91 & 10 & 18.44 & 29.74 \\
\hline
\end{tabular}

$125 \mathrm{GeV}$ may be due to a signal overlap of both Higgs bosons, depending on the mass separation and the mass resolution of the experimental analysis. We show the allowed parameter space in the $\left(m_{h}, m_{H}\right)$ and $\left(m_{h}, \sin \alpha\right)$ plane from the updated fit in Fig. 4. The updated signal strength observables in HiggsSignals-1 4.0 yield only marginal improvements in the constrained parameter space, while the updated limits from direct Higgs searches are irrelevant in this mass region.

\section{Benchmark scenarios for LHC Run 2}

The benchmark scenarios that are presented in this section are chosen such that they feature the maximally allowed production cross section at the LHC. We first present the benchmark scenarios for the high-mass region, where the light Higgs plays the role of the discovered SM-like Higgs at $125 \mathrm{GeV}$, 
Fig. 4 Parameter space for the intermediate mass region after taking all constraints into account. The color coding follows Fig. 3

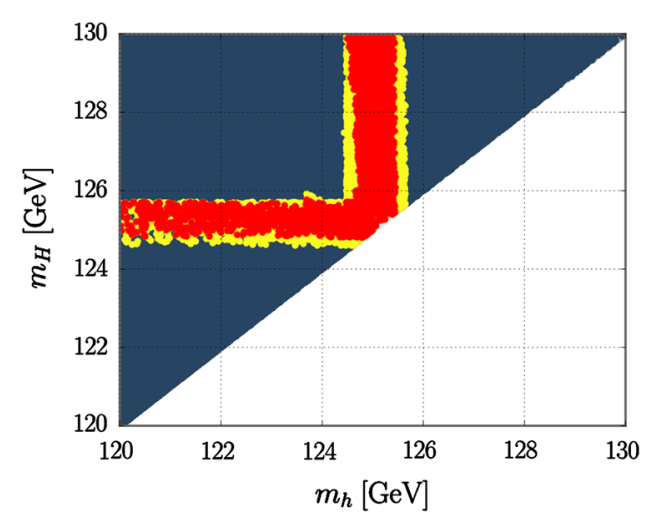

(a) $\left(m_{h}, m_{H}\right)$ plane.

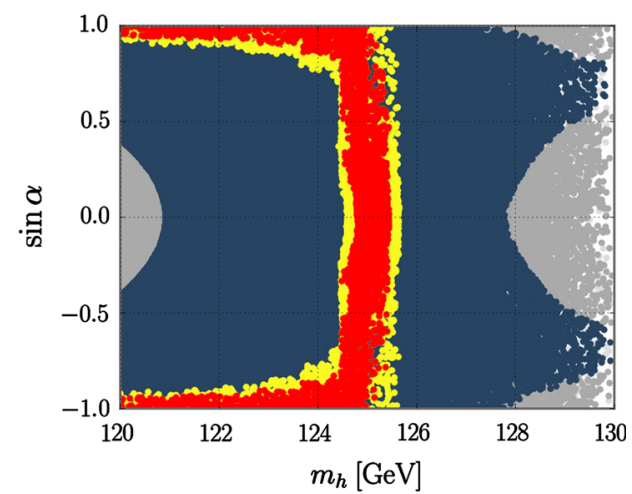

(b) $\left(m_{h}, \sin \alpha\right)$ plane.

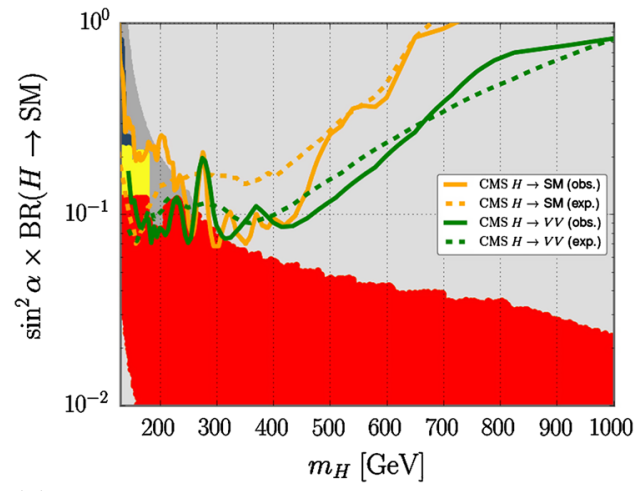

(a) Heavy Higgs signal rate with SM particles in the final state. We display the observed and expected 95\% C.L. limits from the CMS combination of SM Higgs searches [95] as well as from the $H \rightarrow V V(V=W, Z)$ search [66].

Fig. 5 Collider signal rates of the heavy Higgs boson $H$ decaying into SM particles (a) or into two light Higgs bosons, $H \rightarrow h h$, (b), in dependence of the heavy Higgs mass, $m_{H}$. The color coding is the same as in

and then turn to the low-mass range, where the heavy Higgs state is the SM-like Higgs boson. ${ }^{3}$

\subsection{High-mass region}

We distinguish between two different search channels:

- Higgs decays into SM particles: Maximizing the production cross section corresponds to maximizing the parameter [29]

$$
\kappa \equiv \frac{\sigma}{\sigma_{\mathrm{SM}}} \times \mathrm{BR}(H \rightarrow \mathrm{SM})=\sin ^{4} \alpha \frac{\Gamma_{\mathrm{SM}, \mathrm{tot}}}{\Gamma_{\mathrm{tot}}} .
$$

\footnotetext{
3 See also Ref. [99] for recent benchmark point suggestions within the complex singlet model.
}

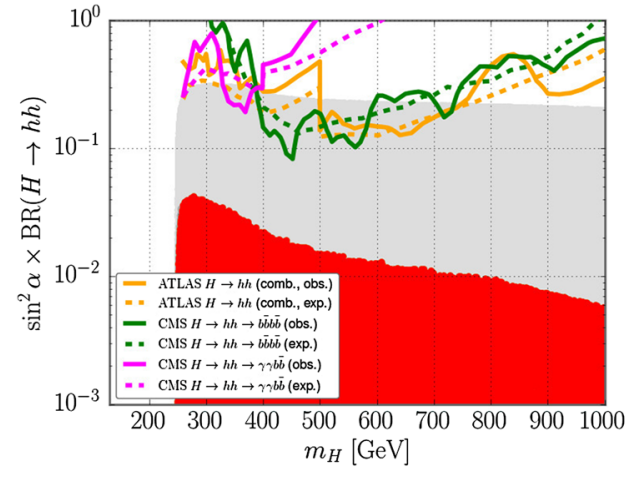

(b) Heavy Higgs signal rate with light Higgs bosons in the final state. We display the current expected and observed $95 \%$ C.L. limits from the ATLAS $H \rightarrow h h$ search (combination of various final states) [67] and CMS $H \rightarrow h h$ searches with $\gamma \gamma b \bar{b}[100]$ and $b \bar{b} b \bar{b}[101]$ final states.

Fig. 3. The rates are normalized to the inclusive SM Higgs production cross section at the corresponding mass value $[23,102,103]$

In general, following Eq. (13), Higgs decays into SM particles follow the hierarchy of the branching ratios of a SM Higgs of the same mass. This, together with the observation that the branching ratio for $H \rightarrow h h$ is $\mathcal{O}(0.2)$ in large parts of the parameter space, translates into the fact that for most of the high-mass region the dominant decay mode is $H \rightarrow W W$.

- Higgs decays into two light Higgs bosons, $H \rightarrow h h$ : Here, the parameter

$$
\kappa^{\prime} \equiv \frac{\sigma}{\sigma_{\mathrm{SM}}} \times \mathrm{BR}(H \rightarrow h h)=\sin ^{2} \alpha \frac{\Gamma_{H \rightarrow h h}}{\Gamma_{\mathrm{tot}}}
$$

is maximized to obtain the largest possible signal yield.

Figure 5 shows the allowed range of these two quantities, after all constraints have been taken into account. For the 
Table 4 Benchmark points for mass ranges where the on-shell decay $H \rightarrow h h$ is kinematically forbidden. Maximal values of $\tan \beta$ were calculated at the maximal mixing angle, and should be applied for consistency reasons

\begin{tabular}{llllll}
\hline$m_{H}[\mathrm{GeV}]$ & $|\sin \alpha|_{\max }$ & $\tan \beta_{\max }$ & $m_{H}[\mathrm{GeV}]$ & $|\sin \alpha|_{\max }$ & $\tan \beta_{\max }$ \\
\hline 130 & 0.42 & 1.79 & 195 & 0.28 & 1.22 \\
135 & 0.38 & 1.73 & 200 & 0.29 & 1.19 \\
140 & 0.36 & 1.69 & 210 & 0.28 & 1.14 \\
145 & 0.35 & 1.62 & 215 & 0.33 & 1.12 \\
150 & 0.34 & 1.57 & 220 & 0.34 & 1.10 \\
160 & 0.36 & 1.49 & 230 & 0.35 & 1.05 \\
180 & 0.30 & 1.32 & 235 & 0.31 & 1.03 \\
185 & 0.27 & 1.28 & 240 & 0.28 & 1.00 \\
190 & 0.29 & 1.26 & 245 & & 0.98 \\
\hline
\end{tabular}

Table 5 Maximal and minimal allowed branching ratios of the decay $H \rightarrow h h$, taken at the maximally allowed value of $|\sin \alpha|$. Note that minimal values for the $\operatorname{BR}(H \rightarrow h h)$ stem from $\sin \alpha \leq 0$

\begin{tabular}{llllllll}
\hline$m_{H}[\mathrm{GeV}]$ & $|\sin \alpha|_{\max }$ & $B R_{\min }^{H \rightarrow h h}$ & $B R_{\max }^{H \rightarrow h h}$ & $m_{H}[\mathrm{GeV}]$ & $|\sin \alpha|_{\max }$ & $B R_{\min }^{H \rightarrow h h}$ & $B R_{\max }^{H \rightarrow h h}$ \\
\hline 255 & 0.31 & 0.09 & 0.27 & 430 & 0.25 & 0.19 & 0.30 \\
260 & 0.34 & 0.11 & 0.33 & 470 & 0.24 & 0.19 & 0.28 \\
265 & 0.33 & 0.13 & 0.36 & 520 & 0.23 & 0.19 & 0.26 \\
280 & 0.32 & 0.17 & 0.40 & 590 & 0.22 & 0.19 & 0.25 \\
290 & 0.31 & 0.18 & 0.40 & 665 & 0.21 & 0.19 & 0.24 \\
305 & 0.30 & 0.20 & 0.40 & 770 & 0.20 & 0.19 & 0.23 \\
325 & 0.29 & 0.21 & 0.40 & 875 & 0.19 & 0.19 & 0.22 \\
345 & 0.28 & 0.22 & 0.39 & 920 & 0.18 & 0.19 & 0.22 \\
365 & 0.27 & 0.21 & 0.36 & 975 & 0.17 & 0.19 & 0.21 \\
395 & 0.26 & 0.20 & 0.32 & 1000 & 0.17 & 0.19 & 0.21 \\
\hline
\end{tabular}

Higgs decay channel into SM particles, we see that searches from CMS pose important constraints for $m_{H} \lesssim 400 \mathrm{GeV}$. For the Higgs-to-Higgs decay channel $H \rightarrow h h$, on the other hand, both ATLAS [67] and CMS [100,101] searches are not yet sensitive enough to exclude points that are not already in conflict with other constraints.

We quantify the benchmark scenarios for both signal channels in this regime by considering the maximally allowed mixing angle together with the maximal and minimal branching ratio for the decay $H \rightarrow h h$, respectively. While these maximal and minimal points define benchmark points, all $\mathrm{BR}(H \rightarrow h h)$ values in between are in principle allowed. Therefore, an interpolation between the minimal and maximal values defines a higher-dimensional benchmark scenario (benchmark slope or plane), where the additional third parameter (cf. Eqs. (17)-(20)) is floating.

We furthermore distinguish scenarios for which the $H \rightarrow$ $h h$ on-shell decay mode is kinematically allowed or forbidden. As we neglect all other triple and quartic Higgs selfcouplings apart from $\mu^{\prime}$, and work in the on-shell approximation, $\tan \beta$ only influences the collider phenomenology for regions in parameter space where the decay $H \rightarrow h h$ is kinematically allowed, i.e. for heavy Higgs masses $m_{H} \geq$ $2 m_{h} \approx 250 \mathrm{GeV}$. For lower masses $\tan \beta$ is irrelevant for the phenomenology considered here. However, to be consistent, we recommend to still keep the values within the respective parameter regions allowed by perturbativity and perturbative unitarity.

Benchmark scenarios for both cases are given in Tables 4 and 5, respectively. Parameter ranges which are not explicitly listed can to a first approximation be linearly interpolated.

In addition, we also list exemplary benchmark points for this mass region in Tables 6 and 7, where we additionally give the predictions for other relevant decay modes. Whenever kinematically accessible, we provide two benchmark points for every heavy Higgs mass, representing the maximal and minimal branching ratio for the $H \rightarrow h h$ decay, respectively. ${ }^{4}$ The mixing angle is always chosen such that the production rate of the additional scalar is maximized.

\subsection{Low-mass region}

For the case that the heavier Higgs boson is taken to be the discovered SM-like Higgs boson with $m_{H} \sim 125 \mathrm{GeV}$, $|\sin \alpha|=1$ corresponds to the SM limit, and deviations

\footnotetext{
${ }^{4}$ Electroweak corrections to the decay $H \rightarrow h h$ have been presented for some of these benchmark points in Ref. [54].
} 
Table 6 Benchmark scenarios for the high-mass region for fixed masses and $|\sin \alpha|$, floating $\tan \beta$ (between scenarios $a$ and $b$ ). Reference production cross sections have been taken from the upcoming CERN Yellow Report 4 by the LHC Higgs Cross Section Working Group [104]

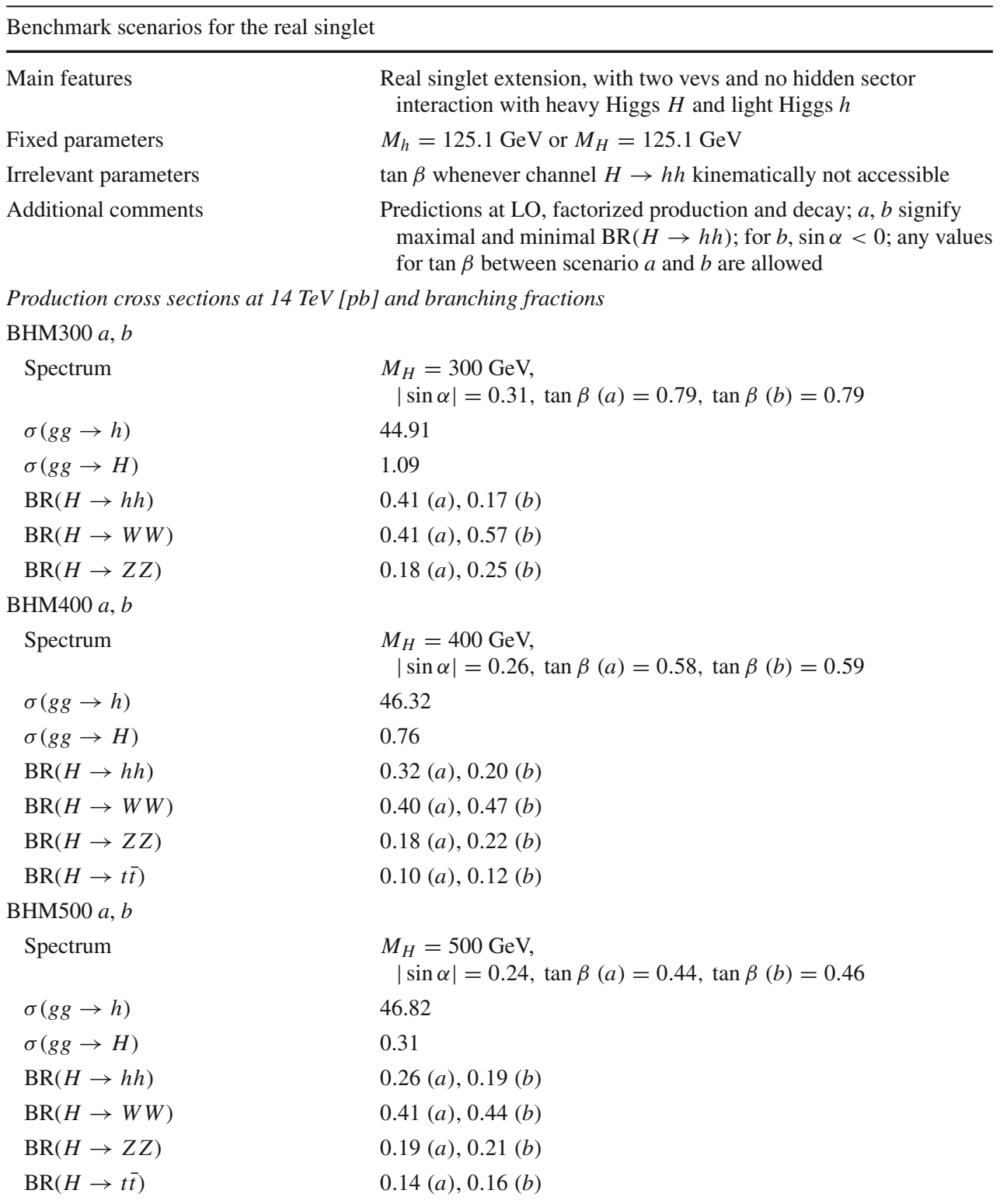

from this value parametrize the new physics contributions. As in the high-mass region, the following channels are interesting:

- Direct production of the lighter Higgs state $h$ and successive decay into SM particles,

- Decay of the SM-like Higgs boson $H$ into the lighter Higgs states, $H \rightarrow h h$.

For the direct production of the light Higgs state smaller $|\sin \alpha|$ values are of interest, as the cross section scales with $\cos ^{2} \alpha$. We provide the minimally allowed values for $|\sin \alpha|$ in Table 2. Table 3 lists the respective direct production cross sections at 8 and $14 \mathrm{TeV}$. These values can directly be used as benchmark scenarios for collider searches for direct light Higgs production.

For the second channel-the decay of the SM-like Higgs into two lighter Higgs states-we list maximal branching ratios for the decay $H \rightarrow h h$ in Table 8 . As long as the decay $H \rightarrow h h$ is kinematically accessible, the maximal value of its branching ratio, $\operatorname{BR}(H \rightarrow h h) \simeq 0.259$, is not dependent on the light Higgs mass. The lighter Higgs bosons then decay further according to the branching ratios of a SM Higgs of the respective mass. A first experimental search of this signature with the light Higgs boson decaying into $\tau$ lepton pairs in the mass range $m_{h} \in[5,15] \mathrm{GeV}$ has already been performed by the CMS experiment [93]. 
Table 7 Benchmark scenarios for the high-mass region for fixed masses and $|\sin \alpha|$, floating $\tan \beta$ (between scenarios $a$ and $b$ ). Reference production cross sections have been taken from the upcoming CERN Yellow Report 4 by the LHC Higgs Cross Section Working Group [104]

Production cross sections at $14 \mathrm{TeV}[\mathrm{pb}]$ and branching fractions (continued)

BHM600 $a, b$

$\begin{array}{ll}\text { Spectrum } & M_{H}=600 \mathrm{GeV}, \\ & |\sin \alpha|=0.22, \tan \beta(a)= \\ \sigma(g g \rightarrow h) & 47.28 \\ \sigma(g g \rightarrow H) & 0.12 \\ \mathrm{BR}(H \rightarrow h h) & 0.25(a), 0.19(b) \\ \mathrm{BR}(H \rightarrow W W) & 0.41(a), 0.45(b) \\ \mathrm{BR}(H \rightarrow Z Z) & 0.21(a), 0.22(b) \\ \mathrm{BR}(H \rightarrow t \bar{t}) & 0.13(a), 0.14(b) \\ \mathrm{BHM} 700 a, b & \\ \mathrm{Spectrum} & M_{H}=700 \mathrm{GeV}, \\ & |\sin \alpha|=0.21, \tan \beta(a)= \\ \sigma(g g \rightarrow h) & 0.31, \tan \beta(b)=0.32 \\ \sigma(g g \rightarrow H) & 47.49 \\ \mathrm{BR}(H \rightarrow h h) & 0.050 \\ \mathrm{BR}(H \rightarrow W W) & 0.24(a), 0.19(b) \\ \mathrm{BR}(H \rightarrow Z Z) & 0.44(a), 0.47(b) \\ \mathrm{BR}(H \rightarrow t \bar{t}) & 0.22(a), 0.23(b) \\ \mathrm{BHM} 800 a, b & 0.10(a), 0.11(b) \\ \mathrm{Spectrum} & \\ \sigma(g g \rightarrow h) & \\ \sigma(g g \rightarrow H) & M_{H}=800 \mathrm{GeV},|\sin \alpha|= \\ \mathrm{BR}(H \rightarrow h h) & 0.2, \tan \beta(a)=0.25, \tan \beta(b)=0.27 \\ \mathrm{BR}(H \rightarrow W W) & 47.69 \\ \mathrm{BR}(H \rightarrow Z Z) & 0.022 \\ \mathrm{BR}(H \rightarrow t \bar{t}) & 0.23(a), 0.19(b) \\ \mathrm{BHM} 200 & 0.46(a), 0.48(b) \\ \mathrm{Spectrum} & 0.23(a), 0.24(b) \\ \sigma(g g \rightarrow h) & 0.08(a), 0.09(b) \\ \sigma(g g \rightarrow H) & \\ \mathrm{BR}(H \rightarrow \mathrm{SM}) & \\ & \\ & \\ & \\ & \end{array}$

Table 8 Maximal branching ratios for $H \rightarrow h h$. This BR can always be zero for the choice $\tan \beta=-\cot \alpha$

\begin{tabular}{lll}
\hline$m_{h}[\mathrm{GeV}]$ & $\sin \alpha$ & $B R_{\max }^{H \rightarrow h h}$ \\
\hline 60 & 0.9996 & 0.259 \\
50 & 0.9999 & 0.259 \\
40 & 0.9999 & 0.259 \\
30 & 0.9999 & 0.259 \\
20 & 0.9998 & 0.259 \\
10 & 0.9999 & 0.259 \\
\hline
\end{tabular}

Table 9 Low-mass benchmark scenarios for the Higgs-to-Higgs decay signature for fixed masses and $|\sin \alpha|$, floating $\tan \beta$ (between scenarios $a$ and $b$ ). In scenario $b$ we have $\tan \beta=-\cot \alpha$. The $|\sin \alpha|$ values have been optimized for scenario $a$, which in turn leads to a suppression of direct production for the lighter state. For direct production of the lighter scalar, the parameters in Tables 2 and 3 should be used. For BHM50 - BHM10, the production cross section for the SM like Higgs is $\sigma(g g \rightarrow H)=49.66 \mathrm{pb}$. Reference production cross sections have been taken from the upcoming CERN Yellow Report 4 by the LHC Higgs Cross Section Working Group [104]

\begin{tabular}{|c|c|}
\hline \multicolumn{2}{|l|}{ BHM60 $a, b$} \\
\hline Spectrum & $\begin{array}{l}M_{h}=60 \mathrm{GeV},|\sin \alpha|=0.9997, \tan \beta(a)= \\
3.48, \tan \beta(b)=0.025\end{array}$ \\
\hline$\sigma(g g \rightarrow h)$ & 0.10 \\
\hline$\sigma(g g \rightarrow H)$ & 49.65 \\
\hline $\mathrm{BR}(H \rightarrow h h)$ & $0.26(a), 0(b)$ \\
\hline $\mathrm{BR}(H \rightarrow \mathrm{SM})$ & Rescaled by $0.74(a)$, as in SM $(b)$ \\
\hline \multicolumn{2}{|l|}{ BHM50 $a, b$} \\
\hline Spectrum & $\begin{array}{l}M_{h}=50 \mathrm{GeV},|\sin \alpha|=0.9998, \tan \beta(a)= \\
3.25, \tan \beta(b)=0.020\end{array}$ \\
\hline$\sigma(g g \rightarrow h)$ & 0.098 \\
\hline $\mathrm{BR}(H \rightarrow h h)$ & $0.26(a), 0(b)$ \\
\hline $\mathrm{BR}(H \rightarrow \mathrm{SM})$ & Rescaled by $0.74(a)$, as in SM $(b)$ \\
\hline \multicolumn{2}{|l|}{ BНM40 $a, b$} \\
\hline Spectrum & $\begin{array}{l}M_{h}=40 \mathrm{GeV},|\sin \alpha|=0.9998, \tan \beta(a)= \\
3.13, \tan \beta(b)=0.020\end{array}$ \\
\hline$\sigma(g g \rightarrow h)$ & 0.16 \\
\hline $\mathrm{BR}(H \rightarrow h h)$ & $0.26(a), 0(b)$ \\
\hline $\mathrm{BR}(H \rightarrow \mathrm{SM})$ & Rescaled by $0.74(a)$, as in SM $(b)$ \\
\hline \multicolumn{2}{|l|}{ ВНМ30 $a, b$} \\
\hline Spectrum & $\begin{array}{l}M_{h}=30 \mathrm{GeV},|\sin \alpha|=0.9998, \tan \beta(a)= \\
3.16, \tan \beta(b)=0.020\end{array}$ \\
\hline$\sigma(g g \rightarrow h)$ & 0.31 \\
\hline $\mathrm{BR}(H \rightarrow h h)$ & $0.26(a), 0(b)$ \\
\hline $\mathrm{BR}(H \rightarrow \mathrm{SM})$ & Rescaled by $0.74(a)$, as in SM $(b)$ \\
\hline \multicolumn{2}{|l|}{ ВНM20 $a, b$} \\
\hline Spectrum & $\begin{array}{l}M_{h}=20 \mathrm{GeV},|\sin \alpha|=0.9998, \tan \beta(a)= \\
3.23, \tan \beta(b)=0.020\end{array}$ \\
\hline$\sigma(g g \rightarrow h)$ & 0.90 \\
\hline $\mathrm{BR}(H \rightarrow h h)$ & $0.26(a), 0(b)$ \\
\hline $\mathrm{BR}(H \rightarrow \mathrm{SM})$ & Rescaled by $0.74(a)$, as in SM $(b)$ \\
\hline \multicolumn{2}{|l|}{ BHM10 $a, b$} \\
\hline Spectrum & $\begin{array}{l}M_{h}=10 \mathrm{GeV},|\sin \alpha|=0.9998, \tan \beta(a)= \\
3.29, \tan \beta(b)=0.020\end{array}$ \\
\hline$\sigma(g g \rightarrow h)$ & 2.98 \\
\hline $\mathrm{BR}(H \rightarrow h h)$ & $0.26(a), 0(b)$ \\
\hline $\mathrm{BR}(H \rightarrow \mathrm{SM})$ & Rescaled by $0.74(a)$, as in SM $(b)$ \\
\hline
\end{tabular}

We present benchmark points for fixed masses in Table 9. Here, $|\sin \alpha|$ values closer to unity are needed in order to obtain maximal branching ratios for this channel, which in turn leads to the reduction of direct production for the lighter state by almost an order of magnitude with respect to the val- 
ues presented in Table 3. Again, we recommend to scan over $\tan \beta$ between the values of scenario $a$ and $b$ (thus defining a higher-dimensional benchmark scenario) in order to obtain a range of possible branching ratios.

\section{Conclusions}

In this paper we have revisited and updated the constraints on the parameter space of the real scalar singlet extension of the SM. In comparison with the previous results presented in Ref. [38], the most important improvements have been made in the constraints from new results in LHC searches for a heavy Higgs boson decaying into vector boson final states, as well as from the ATLAS and CMS combination of the signal strength of the discovered Higgs state. We found that these modify our previous findings in the mass range $130 \mathrm{GeV} \leq m_{H} \leq 250 \mathrm{GeV}$, where now the direct Higgs searches as well as the ATLAS and CMS signal strength combination render the strongest constraints on the parameter space.

Based on these updated results, we have provided benchmark scenarios for both the high-mass and the low-mass regions for upcoming LHC searches. Hereby, we pursued the philosophy of selecting those points which feature a maximal discovery potential in a dedicated collider search of the corresponding signature. We provided predictions of production cross sections for the LHC at $14 \mathrm{TeV}$, and supplemented these with information as regards the branching fractions of the relevant decay modes. We encourage the experimental collaborations to make use of these benchmark scenarios in the current and upcoming LHC runs.

Acknowledgments We thank S. Dawson, C. Englert, M. Gouzevitch, S. Heinemeyer, I. Lewis, A. Nikitenko, M. Sampaio, R. Santos, M. Slawinska, and D. Stoeckinger for useful discussions, as well as G. Chalons, D. Lopez-Val, and G.M. Pruna for fruitful collaboration on earlier related work. TS is supported in parts by the U.S. Department of Energy grant number DE-SC0010107 and a Feodor-Lynen research fellowship sponsored by the Alexander von Humboldt foundation.

Open Access This article is distributed under the terms of the Creative Commons Attribution 4.0 International License (http://creativecomm ons.org/licenses/by/4.0/), which permits unrestricted use, distribution, and reproduction in any medium, provided you give appropriate credit to the original author(s) and the source, provide a link to the Creative Commons license, and indicate if changes were made.

Funded by SCOAP $^{3}$.

\section{References}

1. P.W. Higgs, Phys. Lett. 12, 132 (1964)

2. P.W. Higgs, Phys. Rev. Lett. 13, 508 (1964)

3. F. Englert, R. Brout, Phys. Rev. Lett. 13, 321 (1964)

4. G. Guralnik, C. Hagen, T. Kibble, Phys. Rev. Lett. 13, 585 (1964)

5. T. Kibble, Phys. Rev. 155, 1554 (1967)
6. G. Aad et al. (ATLAS, CMS), Phys. Rev. Lett. 114, 191803 (2015). arXiv: 1503.07589

7. R. Schabinger, J.D. Wells, Phys. Rev. D 72, 093007 (2005). arXiv:hep-ph/0509209

8. B. Patt, F. Wilczek (2006). arXiv:hep-ph/0605188

9. V. Barger, P. Langacker, M. McCaskey, M.J. Ramsey-Musolf, G. Shaughnessy, Phys. Rev. D 77, 035005 (2008). arXiv:0706.4311

10. G. Bhattacharyya, G.C. Branco, S. Nandi, Phys. Rev. D 77 , 117701 (2008). arXiv:0712.2693

11. S. Dawson, W. Yan, Phys. Rev. D 79, 095002 (2009). arXiv:0904.2005

12. S. Bock, R. Lafaye, T. Plehn, M. Rauch, D. Zerwas et al., Phys. Lett. B 694, 44 (2010). arXiv: 1007.2645

13. P.J. Fox, D. Tucker-Smith, N. Weiner, JHEP 1106, 127 (2011). arXiv: 1104.5450

14. C. Englert, T. Plehn, D. Zerwas, P.M. Zerwas, Phys. Lett. B 703, 298 (2011). arXiv:1106.3097

15. C. Englert, J. Jaeckel, E. Re, M. Spannowsky, Phys. Rev. D 85, 035008 (2012). arXiv:1111.1719

16. B. Batell, S. Gori, L.-T. Wang, JHEP 1206, 172 (2012). arXiv: 1112.5180

17. C. Englert, T. Plehn, M. Rauch, D. Zerwas, P.M. Zerwas, Phys. Lett. B 707, 512 (2012). arXiv:1112.3007

18. R.S. Gupta, J.D. Wells, Phys. Lett. B 710, 154 (2012). arXiv: 1110.0824

19. M.J. Dolan, C. Englert, M. Spannowsky, Phys. Rev. D 87, 055002 (2013). arXiv:1210.8166

20. D. Bertolini, M. McCullough, JHEP 1212, 118 (2012). arXiv: 1207.4209

21. B. Batell, D. McKeen, M. Pospelov, JHEP 1210, 104 (2012). arXiv: 1207.6252

22. D. Lopez-Val, T. Plehn, M. Rauch, JHEP 1310, 134 (2013). arXiv: 1308.1979

23. S. Heinemeyer et al. (The LHC Higgs Cross Section Working Group) (2013). arXiv:1307.1347

24. R.S. Chivukula, A. Farzinnia, J. Ren, E.H. Simmons, Phys. Rev. D 88, 075020 (2013). arXiv:1307.1064

25. C. Englert, M. McCullough, JHEP 1307, 168 (2013). arXiv: 1303.1526

26. B. Cooper, N. Konstantinidis, L. Lambourne, D. Wardrope, Phys. Rev. D 88, 114005 (2013). arXiv: 1307.0407

27. C. Caillol, B. Clerbaux, J.-M. Frere, S. Mollet, Eur. Phys. J. Plus 129, 93 (2014). arXiv:1304.0386

28. R. Coimbra, M.O. Sampaio, R. Santos, Eur. Phys. J. C 73, 2428 (2013). arXiv:1301.2599

29. G.M. Pruna, T. Robens, Phys. Rev. D 88, 115012 (2013). arXiv: 1303.1150

30. S. Dawson, A. Gritsan, H. Logan, J. Qian, C. Tully et al. (2013). arXiv: 1310.8361

31. L. Basso, O. Fischer, J.J. van Der Bij, Phys. Lett. B 730, 326 (2014). arXiv:1309.6086

32. D. Lopez-Val, T. Robens, Phys. Rev. D 90, 114018 (2014). arXiv: 1406.1043

33. C. Englert, M. Spannowsky, Phys. Rev. D 90, 053003 (2014). arXiv: 1405.0285

34. C. Englert, Y. Soreq, M. Spannowsky, JHEP 05, 145 (2015). arXiv: 1410.5440

35. C.-Y. Chen, S. Dawson, I.M. Lewis, Phys. Rev. D 91, 035015 (2015). arXiv: 1410.5488

36. D. Karabacak, S. Nandi, S.K. Rai, Phys. Lett. B 737, 341 (2014). arXiv: 1405.0476

37. S. Profumo, M.J. Ramsey-Musolf, C.L. Wainwright, P. Winslow, Phys. Rev. D 91, 035018 (2015). arXiv:1407.5342

38. T. Robens, T. Stefaniak, Eur. Phys. J. C 75, 104 (2015). arXiv: 1501.02234 
39. V. Martfn Lozano, J.M. Moreno, C.B. Park, JHEP 08, 004 (2015). arXiv: 1501.03799

40. A. Falkowski, C. Gross, O. Lebedev, JHEP 05, 057 (2015). arXiv:1502.01361

41. G. Ballesteros, C. Tamarit, JHEP 09, 210 (2015). arXiv: 1505.07476

42. D. Buttazzo, F. Sala, A. Tesi, JHEP 11, 158 (2015). arXiv: 1505.05488

43. S. Banerjee, M. Mitra, M. Spannowsky, Phys. Rev. D 92, 055013 (2015). arXiv: 1506.06415

44. T. Corbett, O.J.P. Eboli, M.C. Gonzalez-Garcia, Phys. Rev. D 93, 015005 (2016). arXiv:1509.01585

45. A. Tofighi, O.N. Ghodsi, M. Saeedhoseini, Phys. Lett. B 748, 208 (2015). arXiv: 1510.00791

46. C.-Y. Chen, Q.-S. Yan, X. Zhao, Y.-M. Zhong, Z. Zhao, Phys. Rev. D 93, 013007 (2016). arXiv: 1510.04013

47. S.I. Godunov, A.N. Rozanov, M.I. Vysotsky, E.V. Zhemchugov, Eur. Phys. J. C 76, 1 (2016). arXiv: 1503.01618

48. M. Duch, B. Grzadkowski, M. McGarrie, JHEP 09, 162 (2015). arXiv: 1506.08805

49. Z.-W. Wang, T.G. Steele, T. Hanif, R.B. Mann (2015). arXiv: 1510.04321

50. N. Bernal, X. Chu, JCAP 1601, 006 (2016). arXiv:1510.08527

51. S. Ghosh, A. Kundu, S. Ray (2015). arXiv: 1512.05786

52. M.J. Dolan, J.L. Hewett, M. KrSmer, T.G. Rizzo (2016). arXiv:1601.07208

53. S. Kanemura, M. Kikuchi, K. Yagyu, Nucl. Phys. B 907, 286 (2016). arXiv: 1511.06211

54. F. Bojarski, G. Chalons, D. Lopez-Val, T. Robens, JHEP 02, 147 (2016). arXiv: 1511.08120

55. E. Maina, JHEP 06, 004 (2015). arXiv:1501.02139

56. N. Kauer, C. O’Brien, Eur. Phys. J. C 75, 374 (2015). arXiv: 1502.04113

57. C. Englert, I. Low, M. Spannowsky, Phys. Rev. D 91, 074029 (2015). arXiv: 1502.04678

58. A. Ballestrero, E. Maina, JHEP 01, 045 (2016). arXiv: 1506.02257

59. S. Dawson, I.M. Lewis, Phys. Rev. D 92, 094023 (2015). arXiv: 1508.05397

60. L. Basso, S. Moretti, G.M. Pruna, Phys. Rev. D 82, 055018 (2010). arXiv: 1004.3039

61. M.J. Strassler, K.M. Zurek, Phys. Lett. B 651, 374 (2007). arXiv:hep-ph/0604261

62. M.J. Strassler, K.M. Zurek, Phys. Lett. B 661, 263 (2008). arXiv:hep-ph/0605193

63. ATLAS Collaboration (2014). ATLAS-CONF-2014-005

64. ATLAS Collaboration (2014). ATLAS-CONF-2014-010

65. G. Aad et al., ATLAS Collaboration. Phys. Rev. Lett. 113, 171801 (2014). arXiv:1407.6583

66. V. Khachatryan et al. (CMS), JHEP 10, 144 (2015). arXiv: 1504.00936

67. G. Aad et al. (ATLAS), Phys. Rev. D 92, 092004 (2015). arXiv: 1509.04670

68. G. Aad et al. (ATLAS), JHEP 11, 206 (2015). arXiv:1509.00672

69. ATLAS Collaboration (2015). ATLAS-CONF-2015-081

70. G. Aad et al. (ATLAS), Eur. Phys. J. C 76, 45 (2016). arXiv: 1507.05930

71. P. Bechtle, O. Brein, S. Heinemeyer, G. Weiglein, K.E. Williams, Comput. Phys. Commun. 181, 138 (2010). arXiv:0811.4169

72. P. Bechtle, O. Brein, S. Heinemeyer, G. Weiglein, K.E. Williams, Comput. Phys. Commun. 182, 2605 (2011). arXiv:1102.1898
73. P. Bechtle, O. Brein, S. Heinemeyer, O. Stål, T. Stefaniak et al., PoS CHARGED2012, 024 (2012). arXiv:1301.2345

74. P. Bechtle, O. Brein, S. Heinemeyer, O. Stål, T. Stefaniak et al., Eur. Phys. J. C 74, 2693 (2013). arXiv:1311.0055

75. P. Bechtle, S. Heinemeyer, O. Stal, T. Stefaniak, G. Weiglein, Eur. Phys. J. C 75, 421 (2015). arXiv: 1507.06706

76. P. Bechtle, S. Heinemeyer, O. Stål, T. Stefaniak, G. Weiglein, Eur. Phys. J. C 74, 2711 (2014). arXiv:1305.1933

77. P. Bechtle, S. Heinemeyer, O. Stål, T. Stefaniak, G. Weiglein, JHEP 1411, 039 (2014). arXiv: 1403.1582

78. M. Bowen, Y. Cui, J.D. Wells, JHEP 0703, 036 (2007). arXiv:hep-ph/0701035

79. N.D. Christensen, C. Duhr, Comput. Phys. Commun. 180, 1614 (2009). arXiv:0806.4194

80. F. Staub (2008). arXiv:0806.0538

81. F. Staub, Comput. Phys. Commun. 185, 1773 (2014). arXiv: 1309.7223

82. ATLAS and CMS Collaborations (2015). ATLAS-CONF-2015044

83. R.N. Lerner, J. McDonald, Phys. Rev. D 80, 123507 (2009). arXiv:0909.0520

84. G. Altarelli, R. Barbieri, Phys. Lett. B 253, 161 (1991)

85. M.E. Peskin, T. Takeuchi, Phys. Rev. Lett. 65, 964 (1990)

86. M.E. Peskin, T. Takeuchi, Phys. Rev. D 46, 381 (1992)

87. I. Maksymyk, C. Burgess, D. London, Phys. Rev. D 50, 529 (1994). arXiv:hep-ph/9306267

88. M. Baak et al., Gfitter Group. Eur. Phys. J. C 74, 3046 (2014). arXiv: 1407.3792

89. J. Alcaraz et al. (ALEPH Collaboration, DELPHI Collaboration, L3 Collaboration, OPAL Collaboration, LEP Electroweak Working Group) (2006). arXiv:hep-ex/0612034

90. T. Aaltonen et al., CDF Collaboration. Phys. Rev. Lett. 108, 151803 (2012). arXiv:1203.0275

91. V.M. Abazov et al., D0 Collaboration. Phys. Rev. D 89, 012005 (2014). arXiv:1310.8628

92. G. Aad et al. (ATLAS), JHEP 01, 032 (2016). arXiv:1509.00389

93. CMS Collaboration (2015). CMS-PAS-HIG-14-022

94. S. Chatrchyan et al., CMS Collaboration. Phys. Rev. D 89, 092007 (2014). arXiv:1312.5353

95. CMS Collaboration (2012). CMS-PAS-HIG-12-045

96. CMS Collaboration (2013). CMS-PAS-HIG-13-003

97. P. Lebrun, Accelerators at the high-energy frontier: Cern plans, projects and future studies. Talk given at XLIII International Meeting on Fundamental Physics Centro de Ciencias de Benasque Pedro Pascual 12-21, March 2015

98. M. Grazzini, Private communication

99. R. Costa, M. Muehlleitner, M.O.P. Sampaio, R. Santos (2015). arXiv: 1512.05355

100. V. Khachatryan et al. (CMS) (2016). arXiv:1603.06896

101. V. Khachatryan et al. (CMS), Phys. Lett. B 749, 560 (2015). arXiv: 1503.04114

102. S. Dittmaier et al. (LHC Higgs Cross Section Working Group) (2011). arXiv:1101.0593

103. S. Dittmaier, S. Dittmaier, C. Mariotti, G. Passarino, R. Tanaka et al. (2012). arXiv:1201.3084

104. The LHC Higgs Cross Section Working Group (2016), to appear 